fécale montre le danger, et la possibilité de trouver des mierobes de fièvres intestinales.

3. Le nombre total des bactéries ne doit pas dépasser 100.000 bactéries par centimètre cube et les colibacilles ne doivent pas être rencontrés dans un dixième de centimètre cube.

4. Les ouvriers et les distributeurs de l'ice-cream doivent être examinés, afin d'éliminer les porteurs de germes.

\title{
LITTÉRATURE
}

[I] A. Doris Bardesley. Journal Hyg., septembre 1938, 527.

[2] Standard Methods of Milks Analysis. Amer. Pub. H. Assac., 1934.

[3] G. S. WiLson. The Bacteriological Grading of Milk. Med. Res. Council, London, 1935.

[4] M. G. El-Gheriany. Putrefaetive Bacteria and their Detrimental Effect on the Hygienic State of Milk. Intern. Tierarztlicher Kongress, Zurich, 1938.

[5] D. H. Bergey. Manual of Determinative Bacteriology, 1939.

\section{LA CASÉINE ET SES DÉRIVÉS PLASTIQUES (1)}

\author{
par \\ JEANNE BRIGANDO, \\ Chargée de Recherches à la Recherche Scientifique
}

La caséine acide, ou caséine proprement dite, résulte de la précipitation du lait par un acide. La caséine-présure, obtenue en ajoutant de la présure au lait, est constituée par du paracaséinate de calcium et des phosphates de calcium. La caséine est une phosphoprotéine, sensible aux moindres traitements physiques, chimiques et biochimiques. Sa structure permet d'expliquer sa propriété de devenir plastique. La caséine-présure est surtout utilisée pour la préparation des matières plastiques, la caséine acide pour l'obtention des colles et des textiles de caséine.

La caséine est une protéine caractéristique de la sécrétion lactée. On la trouve dans tous les laits. Un litre de lait de vache renferme en moyenne $30 \mathrm{gr}$. de caséine, en suspension sous forme de caséinate calcique. Pratiquement, la caséine est retirée du lait de vache écrémé et doit être considérée comme un sous-produit de l'industrie laitière. Elle a de nombreuses applications industrielles par suite de sa propriété de devenir plastique sous l'influence de certains agents physiques et chimiques.

(1) Communication faite aux Journées des Matières plastiques et des Résines synthétiques, à la Maison de la chimie, à Paris, le 13 juin 1939. 
En réalité, l'industrie dispose, sous le même nom de caséine, de deux produits différents par leur constitution chi mique : la caséine acide ou caséine proprement dite et la caséine-présure. Cet état de chose amène des confusions regrettables.

\section{$1^{\circ}$ La caséine acide.}

Par acidification du lait, la caséine est libérée de son caséinate calcique. Elle précipite lorsque le $p \mathrm{H}$ du milieu atteint 4,7 , qui est son point iso-électrique. Ce phénomène a lieu par addition d'un acide quelconque au lait ou sous l'influence de l'acide lactique qui se forme dans le lait par suite de la fermentation lactique qui s'y développe naturellement avec le temps. On obtient ainsi la caséine acide, qui, séchée, est une matière blanche pouvant être facilement. pulvérisée, se gonflant dans l'eau sans se dissoudre, soluble dans les acides et dans les alcalis. Théoriquement, elle doit être sans charge minérale.

\section{$2^{\circ}$ La caséine-présure.}

$\mathrm{Si}$, à un lait de vache à la température de $30^{\circ}$ environ, on ajoute de la présure, on obtient un caillé d'aspect analogue au caillé lactique, mais chimiquement différent. La caséine-présure ainsi obtenue renferme $7,5 \%$ environ de matières minérales ; elle est constituée par du paracaséinate de ealcium et des phosphates de calcium. Le paracaséinate de calcium est un easéinate de calcium ayant subi l'action de la présure. Elle est insoluble dans l'eau, se dissout dans les acides, mais se dissout très mal dans les alcalis.

On peut envisager le produit sans phosphates de caleium, qui est, en effet, un nouveau dérivé, le paracaséinate de calcium, dont la charge minérale oseille autour de $4 \%$, puis le produit complètement déminéralisé, la paracaséine. Cette paracaséine, comme la caséine acide, se dissout dans les solutions alcalines, mais elle se différencie de cette dernière :

$1^{\circ}$ Par sa sensibilité vis-à-vis des sels calciques. En effet, une suspension de caséinate et de calcium modifiée par la présure précipite pour de très faibles doses de chlorure de calcium comparativement au même caséinate de calcium non emprésuré. C'est Hammarsten qui le premier a mis le phénomène en évidence; ces résultats ont été confirmés par les travaux de Ch. Porcher.

$2^{\circ}$ Par l'irréversibilité de la réaction biochimique labique. On ne connaît aucune réaction qui permette de transformer la paracaséine en caséine. L'empreinte de la présure est indélébile. 


\section{I. - CONSIDÉRATIONS GENÉRALES SUR LES MÉTHODES INDUSTRIELLES DE PRÉPARATION DES CASÉINES}

\section{$1^{\circ}$ La caséine acide.}

La caséine acide est obtenue industriellement :

$1^{\circ}$ Par fermentation lactique (caséine lactique) ;

$2^{\circ}$ Par précipitation par les acides.

1o Par fermentation lactique. - La préparation des caséines lactiques est soumise à beaucoup d'aléas. L'hiver, par suite de la température trop basse, les ferments lactiques se développent mal et. le lait arrive péniblement à cailler. Il est souvent chauffé avant que la coagulation soit complète, c'est-à-dire que le caséinate calcique soit complètement libéré de tout son calcium. Dans ce cas, il n'est pas surprenant que le produit obtenu soit très chargé en matières minérales. L'été, d'autres fermentations que la fermentation lactique peuvent entrer en ligne de compte, et les caséines résultantes sont altérées.

Cette méthode, la plus répandue en France, donne, en somme, des caséines irrégulières dans leur composition.

$2^{\circ}$ Par précipitation par un acide. - Les procédés généralement utilisés consistent à ajouter un acide à un lait chauffé. Nous avons montré, dans une étude antérieure, qu'il était impossible, dans ces conditions, d'arriver à une décalcification complète du produit. La teneur en matière minérale varie avec la température à laquelle on opère. C'est pourquoi nous avons cherché à mettre au point une méthode peu coûteuse, ne demandant pas d'installation spéciale dans le but d'obtenir un produit aussi pur que celui préparé en laboratoire. Le mode opératoire auquel nous nous sommes arrêté a fait l'objet d'une première publication le 27 novembre 1930 (1). Il résulte d'un ensemble de recherches (2). où chacun des facteurs susceptibles d'intervenir : température du milieu, $p \mathrm{H}$, agitation, etc., a été étudié indépendamment des autres, que la température du lait au moment de l'addition de l'acide a une grosse importance. Nous avons montré, d'autre part, qu'il est possible, du fait. que le lait est un milieu tampon, de descendre pour précipiter la caséine au-dessous de son point isoélectrique, sans la solubiliser. Le $p H$ atteint au moment de la précipitation peut avoir une influence sur la qualité du produit et, par suite, retentir sur ses applications. La caséine obtenue au $p \mathrm{H} 4$ a une teneur en cendre très faible de $0,25 \%$ et se pulvérise très facilement à la main.

Nous donnons ci-joint ce procédé de préparation :

Le lait est précipité par un acide à une température aussi basse

(1) Ch. Porcher, J. Brigando, B.F., n 694.032.

(2) J. Brigando, Recherches sur la caséine, thèse, 1933, Ed. “ Le Lait w, Paris. 
que possible, inférieure à $25^{\circ}$, en agitant. La précipitation doit se faire, au point iso-électrique, caractérisé par un $p H=4,7$, plutôt au-dessous, en s'enfonçant dans la zone acide. On peut descendre jusqu'au $p \mathrm{H} 4$ sans diminution du rendement.

La caséine est ensuite cuite dans son sérum à $40^{\circ}-50^{\circ}$ voire $60^{\circ}$ $65^{\circ}$, en élevant graduellement la température. La température de la cuisson, elle aussi, n'est pas négligeable et aura une répercussion sur la qualité du produit.

Le sérum est ensuite décanté. La caséine est lavée à une eau acide froide de $p H 4,7$ en agitant pour disloquer les grains.

\section{2 . La caséine-présure.}

La France a la réputation de préparer, spécialement dans le département de la Charente-Inférieure, la meilleure caséine-présure. Aussi jugeons-nous inutile de parler de cette fabrication, pourtant la plus importante chez nous.

\section{$3^{\circ}$ La paracaséine.}

Le principe de la préparation industrielle que nous avons mise au point consiste à dissoudre la caséine dans un alcali (soude, ammoniaque, chaux) à un $p H \leqslant 7$. Le caséinate chauffé à $30^{\circ}$ est transformé par la présure en paracaséinate, d'où la paracaséine est précipitée par un acide.

\section{$4^{\circ}$ Le paracaséinate de calcium.}

La méthode de préparation consiste à coaguler par la présure un caséinate calcique en présence de sels solubles de calcium.

On fait tout d'abord un caséinate de réaction alcaline constituée par :

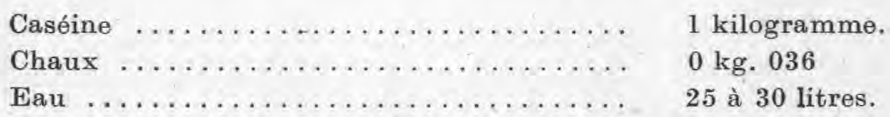

On ramène au $p H \leqslant 7$ par addition d'un acide (acide sulfurique, acide chlorhydrique, acide carbonique ou acide acétique), afin de pouvoir faire agir la présure. On a un liquide homogène, laiteux, constitué par un caséinate calcique et des sels solubles de calcium. Par addition de la présure à la température de $30^{\circ}-40^{\circ}$, ce caséinate caleique coagule. Pour les détails opératoires concernant la préparation de la paracaséine et du paracaséinate de calcium, nous renvoyons le lecteur au travail déjà mentionné (2).

\section{$5^{\circ}$ Le séchage des caséines.}

Les caséines doivent être séchées après leur précipitation si on veut les conserver. Une caséine tant acide que présure renferme 
après son séchage une quantité d'eau qui varie de 6 à $10 \%$ et qui doit être considérée comme une eau de constitution. Une caséine préparée en laboratoire ayant subi plusieurs traitements à l'alcool et à l'éther, renferme $10 \%$ d'eau environ. Si cette eau est éliminée par séchage à $100^{\circ}$, la caséine se dissout diffieilement dans les alcalis. On comprend que, industriellement, un grand soin devra être apporté au séchage. S'il est effectué à une température trop élevée, la surface du grain sera cornée par suite d'une déshydratation trop brutale et la caséine sera modifiée dans sa structure.

Il existe de bons procédés de séchage généralement réservés à la caséine pure, dont la fabrication, tout au moins en France, est plus soignée que celle de la caséine acide. La texture du grain intervient dans cette opération.

\section{II. - LA CASEINE. SA COMPOSITION CHIMIQUE. SA STRUCT URE}

La caséine est une phosphoprotéine. Par hydrolyse, on a isolé la plus grande partie des acides aminés qui entrent dans sa constitution et qui sont au nombre de vingt-six (1).

Il résulte de la nature de ces acides aminés que la caséine renferme plus de groupes carboxyles que de groupes aminés. Cela explique pourquoi la caséine a un caractère acide. Sa dissolution dans un alcali correspond à la neutralisation des $\mathrm{COOH}$ libres, puis à la salification des carboxyles liés aux groupes aminés qui sont libérés par hydrolyse. La présence des groupes aminés permet d'expliquer sa solubilité dans les acides. De fortes concentrations acides et alcalines hydrolysent la caséine. Elle n'est relativement stable qu'entre les $p$ H 2,5 et 8,5 .

La caséine ne donne pas de diagrammes de rayons $\mathrm{X}$ caractéristiques. La caséine séchée donne des diagrammes de protéine à l'état $\beta$, stade final de la dénaturation d'après Astbury. On est, par suite, obligé, pour sa structure, de rester dans le domaine des hypothèses en faisant des rapprochements avec les autres protéines et, en particulier, les protéines kératiniques, pour lesquelles on a des données plus précises (diagrammes de fibres relativement nets).

Du fait qu'elle est une protéine, on peut admettre, d'après la théorie de Fischer, qu'elle est formée d'un agrégat de chaînes peptiques résultant de la polymérisation des acides aminés avec élimination d'eau. D'après ABDERHALDEN, les acides aminés seraient sous forme de dicétopipérazides substitués, les anneaux étant reliés les uns aux autres par des válences secondaires. AstBury a pu mettre en évidence, grâce aux rayons $\mathrm{X}$, ces deux formes de structure dans les protéines kératiniques : la forme peptidique et la forme de

(1) Ross AIKen Gortwer, Casein and its industrial applications by E. Sutermeister and F. Browne, 1939, Ed. Reinhold, U.S.A., ch. 2, p. 38. 
dicétopipérazide. L'interprétation des diagrammes conduit aux schémas suivants :

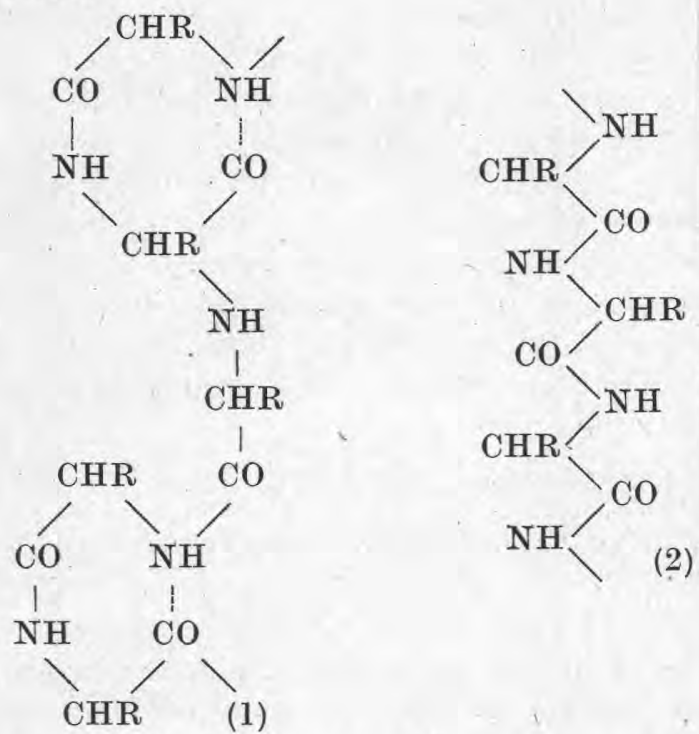

Le schéma (1) correspond à la forme $\alpha$ à chaînes repliées.

Le schéma (2) correspond à la forme $\beta$ ou état étiré.

De nombreuses hypothèses ont été faites sur le mode de liaison des chaînes peptidiques entre elles, pour constituer la micelle des diverses protéines. On peut concevoir que les chaînes longitudinales sont reliées entre elles par des chaînes latérales constituant des sortes de ponts.

Louis Meunier admet, pour les gélatines, que chacun des groupements $\mathrm{CO}$ et $\mathrm{NH}$ des liaisons peptidiques est pourvu de valences secondaires susceptibles d'être utilisées soit pour fixer des molécules d'eau, soit pour souder ensemble les unes aux autres les chaînes peptidiques voisines.

En traitant la caséine avec de l'alcool faiblement chlorhydrique et en précipitant l'extrait par la soude, LINDERSTRom-LANG a séparé de la caséine des fractions différentes entre elles par leur contenu en phosphore et en acides aminés. Ces fractions mélangées peuvent redonner un produit ayant les mêmes propriétés que la caséine originelle (1). Par suite, les chaînes d'acides aminés doivent être considérées comme groupées pour former des composés pouvant être séparés facilement de la caséine.

On peut imaginer tout l'intérêt d'un produit sensible aux

(1) Landerstrom Lang (K.), Z. Physiol. Chem., 176, 76, 1928; C. R. Trav. Lab. Carlsberg, 17, no $9,1929$. 
moindres traitements tant physiques que chimiques. Quand on voudra travailler la caséine, l'action de la température, du $p \mathrm{H}$, de l'eau, ne devra pas être négligée.

Par hydrolyse ménagée, il sera possible de briser certaines de ces chaînes et d'obtenir un grand nombre d'états intermédiaires avant d'arriver à la désintégration totale en acides aminés.

Les diastases, les microorganismes agiront sur les acides aminés de leur choix et d'après l'orientation des chaînes ; les actions biochimiques seront différentes de celles des agents d'hydrolyse chimique toujours plus brutales, mais qui néanmoins peuvent être graduelles. La disposition en chaînes permet d'espérer la possibilité d'arriver à les orienter, ce qui serait un immense progrès pour la fabrication des fibres textiles.

D'après Astbury, il faudrait, par des réactions bien contrôlées, pouvoir briser les chaînes latérales possédant les fonctions chimiques et qui, par suite de réactions entre elles, produisent des replis des chaînes principales qui masquent les caractères essentiels de leur structure.

De ces considérations, il ressort que la caséine est une substance excessivement labile. Elle peut devenir, par contre, très solide sous l'action de certains réactifs chimiques. On peut imaginer que des ponts soient lancés entre les chaînes dans le but de les verrouiller. En bloquant certaines fonctions, de nouveaux composés résistants prennent naissance. C'est ainsi que l'aldéhyde formique, par suite d'une réaction de condensation avec les groupes aminés, forme une matière nouvelle ne se gonflant plus à l'eau, résistant à l'action des acides, des diastases, des micro-organismes. Parmi les produits chimiques susceptibles de former de nouveaux composés avec la caséine, on peut citer les aldéhydes, des corps appelés durcissants tels que les sels de chrome, les aluns, le tannin, etc.

\section{I. - LA PLASTICITÉ}

La caséine acide et la caséine-présure ont la propriété de devenir plastiques, c'est-à-dire de se transformer en une substance compacte pouvant être moulée.

La caséine-présure devient plastique sous l'action de la chaleur et de l'eau sous une certaine pression. Cette plasticité augmente si on déminéralise la caséine-présure. Il est reconnu en fromagerie que les laits donnent des caillés d'autant plus caoutchouteux qu'ils sont plus acides.

La caséine acide doit être au préalable dispersée par un alcali si on veut la transformer en un produit se moulant facilement sous une faible pression. Certains corps tels que des huiles de ricin sulfonées, des alcools supérieurs, des amines de la série grassé ou 
aromatique, etc., rendent la caséine acide et la caséine-présure plus molle et sont appelés des plastifiants.

Nous allons essayer d'interpréter le mode d'action de la chaleur, de l'eau et des plastifiants en empruntant quelques idées au travail de M. Mathieu (1). D'une manière générale, les propriétés plastiques sont toujours observées lorsque, dans une substance, on peut libérer des plans de glissement.

On peut pënser que par l'action de la chaleur, la cohésion entre les chaînes diminue de telle sorte qu'un glissement apparaisse.

L'eau joue un rôle très important. Les expériences faites par Astbury sur la laine ont montré que l'eau absorbée se place entre les chaînes principales dans la direction des chaînes latérales, pouvant causer des altérations des distances réticulaires le long de ces chaînes. Une étude très intéressante de M. Mathieu et de M. Desmaroux sur l'action des vapeurs d'acétone sur les nitrocelluloses permet d'interpréter le rôle des plastifiants. "Tout porte à croire que les chaînes s'écartent pour que puissent se loger entre elles les molécules d'acétone... On a remplacé tous les contacts entre chaînes qui se faisaient par des groupes - $\mathrm{NO}^{2}$ par des contacts entre groupes $-\mathrm{CH}_{3}$. On a créé dans l'intérieur des cristallites entre les chaînes moléculaires les mêmes contacts que ceux qui existent entre plans de glissement des cristaux de paraffines... Grâce à l'introduction de solvants, de gélatinisants, dans les structures, on libère les chaînes moléculaires des liens qu'elles gardent entre elles. On rend possible des glissements entre ces chaînes, la matière devient plastique $» . .$.

\section{$1^{0}$ La plasticité des caséines-présure.}

L'eau, intervenant comme un plastifiant, s'intercalant entre les chaînes, pourrait expliquer pourquoi la caséine-présure est plus plastique que la caséine acide et pourquoi la paracaséine et ses sels de sodium et de calcium, sont eux-mêmes plus plastiques que la caséine-présure. On peut admettre que, sous l'influence diastasique, les valences secondaires reliant les chaînes entre elles sont scindées et saturées par des molécules d'eau. D'autre part, l'action de la présure s'accompagnant d'une action protéolytique, des chaînes sont libérées et de nouveaux plans de glissement peuvent ainsi apparaître.

La paracaséine et ses sels ont, d'autre part, une plus grande faculté de fixer de l'eau que la caséine-présure. Le paracaséinate calcique a une telle tendance à se gonfler dans l'eau qu'il nous fut

(1) Les propriétés élastiques et plastiques des hauts polymères en relation avec leur structure moléculaire (Rev. Gén. Caoutchouc, no 143, juillet-août 1938). 
impossible de le laver lors de sa préparation par le procédé que nous avons indiqué.

La paracaséine peut aussi fixer de l'eau très solidement sur sa trame. Elle doit être pressée immédiatement après sa sortie de la cuve, sinon elle devient "grasse " en terme de métier.

La paracaséine et ses sels plus plastiques que la caséine-présure n'ont pas encore été utilisés dans l'industrie. Ce sont des produits nouveaux en ce sens, et qui pourraient être intéressants soit comme matière plastique, soit pour l'obtention de colles et de fibres textiles. En laboratoire, nous avons pu étirer la paracaséine en fils ténus presque invisibles.

\section{$2^{\circ}$ La plasticité de la caséine acide,}

La caséine acide est moins plastique que la caséine-présure. Elle doit, au préalable, être hydrolysée partiellement par les réactifs alcalins. Ce principe est la base de la préparation des colles et des caséines textiles. On peut penser que l'hydrolyse, par suite de la libération de certains acides aminés, facilite de nouveaux glissements. De plus, à la faveur de ces traitements, de l'eau peut s'intercaler entre les chaînes, agissant comme plastifiant. L'application de l'équilibre de Donnan à un caséinate alcalin, RNa, par exemple, formé d'un ion $\mathrm{R}$ négatif non diffusible et d'un ion Na diffusible, permet d'expliquer une absorption d'eau par le caséinate alcalin.

\section{IV. - LES DÉRIVÉS PLASTIQUES DE LA CASÉINE}

\section{$1^{0}$ Les matières plastiques.}

a) Matière plastique obtenue à partir de la caséine-présure. - En présence de l'eau sous l'action de la chaleur et surtout d'une certaine pression, la caséine-présure se transforme en un produit compact. Les quantités d'eau employées varient de 30 à $45 \%$ environ. L'eau peut être avantageusement remplacée par l'alcool. Les produits plastiques obtenus sous forme de baguettes ou de feuilles, selon les appareils utilisés, sont ensuite placés dans un bain de formol pour être durcis. Il est nécessaire que la solution de formaldéhyde pénètre la totalité de la masse. La durée du traitement varie avec l'épaisseur de cette dernière, d'une semaine à six mois. Il a été suggéré des traitements aux vapeurs de formol, l'emploi de sels de chrome, de tanin, d'aluns, en vue de remplacer le traitement très long des bains de formol.

En 1929, Christensen trouve que l'incorporation de $2 \%$ d'un sel d'aluminium, soluble dans l'eau à la caséine-présure, donne une matière assez rigide pour être travaillée sans être formolée. Ce plastique doux est transformé en boutons qui sont ensuite durcis dans 
un bain de formol, dont la durée d'action est de trois à cinq jours seulement (1).

b) Matière plastique obtenue à partir de la caséine acide. - Le procédé industriel, d'ailleurs peu employé, consiste à disperser la caséine dans une solution alcaline et à la coaguler ensuite par un acide. Le grain obtenu peut se transformer en une matière plastique molle qui est durcie par un bain de formol. Ce produit est plus cassant que celui préparé à partir de la caséine-présure. Nous avons obtenu en laboratoire une matière se moulant facilement à partír d'une caséine lactique très ordinaire par un autre procédé. Le produit résultant est opaque et non cassant.

\section{$2^{\circ}$ Les colles de caséine.}

Les colles de caséine sont très employées pour l'industrie des bois contreplaqués, l'encollage des papiers, l'industrie des cuirs. C'est un chapitre fort important que nous n'avons pas la prétention de traiter ici. Nous empruntons quelques données au riche exposé de Frédérick L. Browne et Don Brouse dans l'ouvrage de Sutermeister et de F. L. Browne.

Une colle est une matière visqueuse capable de se transformer avec le temps en un gel très adhésif sur les surfaces devant être unies.

Actuellement, pour la préparation des colles de caséine, on ajoute 3 à 4 gr. d'eau par gramme de caséine, l'eau contenant 5 à $10 \%$ d'alcali dissous et des sels. Par suite de la forte alcalinité, la caséine est hydrolysée, comme cela est évident par la forte odeur d'ammoniac qu'elle dégage, de sorte que, lorsque la colle a durci, à peu près toute la caséine est transformée en ses produits de clivage, dont la composition est moins connue que celle de la caséine.

En résumé, une colle est une solution d'une viscosité désirable, de caséine acide dans un alcali. On opère avec de fortes concentrations alcalines pour lesquelles l'hydrolyse commence et progresse ensuite très rapidement. Dans ces conditions, les solutions ont une durée de vie relativement courte (quelques heures).

La viscosité des solutions de caséine dans les alcalis varie en fonction de la concentration en caséine et en fonction du $p \mathbf{H}$. Pour des concentrations de $1 \%$ de caséine dans différents alcalis, la viscosité maximum est obtenue pour un $p H$ voisin de 11,5 . Pour des concentrations de $9 \%$ en caséine, la viscosité maximum a lieu à un $p \mathbf{H}$ de 9,2 . Elle décroît ensuite très rapidement pour des $p \mathbf{H}$ supérieurs, exception faite du cas où la base utilisée est l'ammoniaque. Pour les solutions de caséinate d'ammoniaque, le maximum

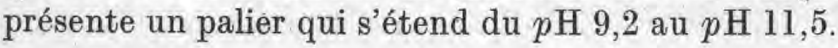

(1) Extrait du « Livre de E. Sutermeister et F. L. Browne „, loc. cit. p. 199. 
L'alcali généralement employé pour la préparation des colles de caséine est la soude. L'ammoniaque est utilisée quand on désire une faible hydrolyse de la protéine. Pour avoir des colles plus résistantes à l'eau, on préfère le caséinate de calcium. Différents types de formules sont donnés par F. Browne et D. Brouse.

\section{$3^{\circ}$ Les textiles de caséine.}

L'emploi de la caséine comme textile a fait l'objet de plusieurs brevets.

En général, le principe consiste à disperser la caséine acide dans un alcali. Après maturation, la solution est passée à travers des filières, et la caséine est précipitée par un bain acide. Elle est ensuite durcie en bain de formol additionné de différents produits.

La maturation est certainement le point le plus délicat de la fabrication. La quantité de soude ajoutée à la caséine est élevée. Dans un de ces procédés, à $110 \mathrm{gr}$. de caséine sont ajoutés $9 \mathrm{gr}$. de soude environ. Pour atteindre le $p$ H 7, il aurait suffi d'utiliser $2 \mathrm{gr} .7$ de soude environ. Par suite, les solutions sont fortement alcalines, La maturation correspond à une hydrolyse partielle de la caséine.

Mais, si l'hydrolyse était trop poussée, la caséine serait complètement scindée en ses acides aminés et aurait perdu ses propriétés plastiques.

Dans un autre procédé, plus récent, la caséine est dissoute dans un alcali caustique. La masse amenée à la consistance voulue après dilution et addition de certains produits chimiques est forcée à travers des filières. La fibre est durcie par un bain contenant du formol et d'autres réactifs.

Aucun procédé industriel n'a jusqu'alors pensé à s'adresser soit au paracaséinate de sodium ou de calcium, soit à la paracaséine.

Ces produits, gonflés à l'eau, pourraient être filés par passage sous pression à travers des filières à une certaine température. La paracaséine pourrait au préalable subir des traitements chimiques dans le but de bloquer soit ses fonctions amines, soit ses fonctions acides.

En laboratoire, nous avons pu obtenir, par un procédé différent, des fils très ténus à peu près invisibles, à partir de la paracaséine.

Ce procédé, non encore publié, comporte les opérations suivantes :

$1^{\circ}$ Dissolution dans de l'eau de chaux à un $p \mathrm{H} \leqslant 7$ d'une caséine sans charge minérale obtenue d'après notre procédé ;

$2^{\circ}$ Attaque par la présure à une température voisine de $30^{\circ}-35^{\circ}$;

$3^{\circ}$ Passage de cette solution à travers des filières ;

$4^{\circ}$ Coagulation par un bain acide ;

$5^{\circ}$ Etirage du fil dans l'eau chaude ;

$6^{\circ}$ Traitement par une solution de formol ou un produit équivalẹnt. 
Si, au lieu d'un caséinate de calcium, nous emprésurons un caséinate de sodium de $p H \leqslant 7$, nous n'obtenons pas, après précipitation par les acides, un produit aussi plastique donnant un fil aussi fin. Les meilleurs résultats sont obtenus avec le caséinate de calcium.

$$
*^{*} *
$$

Des perfectionnements sont continuellement apportés aux méthodes existantes. De gros efforts sont poursuivis actuellement dans tous les pays pour organiser l'industrie de la caséine et de ses dérivés plastiques, mais la France semble, à l'heure actuelle, rester en dehors du mouvement.

Voici un passage que nous extrayons du livre si documenté de Sutermeister et de F. Browne (1) :

"Les industriels européens fabricants de caséine plastique ont, depuis quelques années, reconnu la valeur des associations commerciales. En Grande-Bretagne, elles sont actives et elles ont fait du bon travail commercial et de contrôle de la qualité des produits. Elles ont joué également un rôle éducatif et social. Les associations commerciales sont aussi fortes en Tchécoslovaquie. En Italie, elles sont actives et très fortes, mais elles agissent sous le contrôle gouvernemental, tandis qu'en Allemagne elles ont presque cessé de travailler pour l'industrie en général, mais elles ont organisé certaines spécialités telles que la confection des boutons, etc... En France, le mouvement n'a pas gagné beaucoup d'ampleur. "

Ces dernières considérations se rapportent au côté économique du problème de la caséine et de ses dérivés plastiques. Il serait souhaitable de ne pas perdre de vue l'aspect scientifique et technique de la question. Des résultats vraiment intéressants ne peuvent être obtenus pour des produits aussi sensibles aux actions extérieures qu'après avoir abandonné les méthodes empiriques et que par une collaboration entre la recherche scientifique et l'industrie.

\title{
L'UTILISATION DU SÉRUM : LA FABRICATION DU SUCRE DE LAIT PUR
}

\author{
par \\ G. GENIN \\ Ingénieur Chimiste E. P. C.
}

Nous avons, dans une étude précédente (2), indiqué d'après M. Springer, de la Sheffield By-Products Company, comment on pouvait utiliser d'une façon rationnelle et intéressante les matières solides contenues dans le sérum du lait pour la fabrication d'un

(1) Sutermeister et F. Browne, loc. cit., p. 184.

(2) Le Lait, 1940, n० 198-200, septembre-décembre, p. 510 . 